\title{
Wave observations at the foreshock boundary in the near-Mars space
}

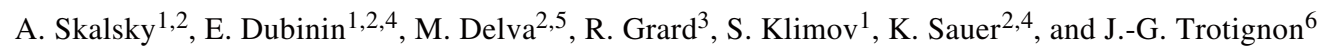 \\ ${ }^{1}$ Space Research Institute, Profsoyuznaya 84/32, 117810, GSP7 Moscow, Russia \\ ${ }^{2}$ International Space Science Institute (ISSI), Hallerstrasse 6, 3012 Bern, Switzerland \\ ${ }^{3}$ Space Science Department, ESA/ESTEC, Postbus 299, 2200 AG Noordwijk, The Netherlands \\ ${ }^{4}$ Max-Planck-Institut für Aeronomie, D-37191 Katlenburg-Lindau, Germany \\ ${ }^{5}$ Institut für Weltraumforschung/OAW, 8010 Graz, Inffeldgasse 12, Austria \\ ${ }^{6}$ Laboratoire de Physique et de Chimie de l'Environnement/CNES, 45071 Orleans Cedex 02, France
}

(Received August 4, 1997; Revised January 19, 1998; Accepted February 20, 1998)

\begin{abstract}
Wave and plasma measurements carried out by the Phobos- 2 spacecraft in the Martian environment indicate that a part of solar wind electrons and ions are reflected and accelerated at the Martian bow shock. These particles streaming back into the solar wind lead to the formation of the foreshock region upstream the planetary bow shock. It is shown that the general structure of the foreshock at Mars is similar to that of the foreshock region upstream the Earth's bow shock. However, the electric emissions observed at frequencies around $100 \mathrm{~Hz}$ at the leading edge of electron foreshock at Mars have been never reported for observations in the similar region at Earth. The burst of these waves occurs before the spacecraft enters the electron foreshock identified with the onset of electron plasma waves and calculations of the magnetic connection to the planetary bow shock.
\end{abstract}

\section{Introduction}

The Plasma Wave System (PWS), flown onboard the Phobos-2 spacecraft, carried out the first investigation of the natural waves in the Martian environment. The PWS experiment measured the electric fields by means of a dipole antenna made of two spheres, each of $10 \mathrm{~cm}$ in diameter, separated by a distance of $1.45 \mathrm{~m}$ (Grard et al., 1989a). The signal collected by the antenna was analyzed with a filter bank consisting of 25 frequency channels and covering a wide frequency range from $0.2 \mathrm{~Hz}$ to $150 \mathrm{kHz}$. A variety of electric field oscillations at different frequencies were detected by the PWS experiment upstream the Martian bow shock (Grard et al., 1989b). High-frequency emissions, broadband electric noise, whistler modes and ULF fluctuations were typically recorded by the wave instrument before the Phobos- 2 spacecraft crossed the bow shock. The enhanced level of magnetic field oscillations in the ULFrange upstream the Martian bow shock was reported by Russell et al. (1990) and Delva and Dubinin (1998). Simultaneous plasma observations have revealed the presence of both ions and electrons reflected from the bow shock and streaming back to the solar wind (Kiraly et al., 1991; Barabash and Lundin, 1991). It has been also shown that plasma and wave observations, carried out upstream the Martian bow shock, are closely linked. Skalsky et al. $(1992,1993)$ have demonstrated that the high frequency emissions at the leading edge of the foreshock are correlated with the enhanced electron fluxes streaming into the solar hemisphere along the magnetic field line. Tarasov et al. (1998) presented the evidence

Copy right (C) The Society of Geomagnetism and Earth, Planetary and Space Sciences (SGEPSS); The Seismological Society of Japan; The Volcanological Society of Japan; The Geodetic Society of Japan; The Japanese Society for Planetary Sciences. that the ULF fluctuations of the magnetic field were observed simultaneously with the appearance of ions reflected at the Martian bow shock and gyrating in the upstream region (Dubinin et al., 1994, 1995). All these phenomena have allowed to postulate the presence of a well developed foreshock upstream the Martian bow shock which is formally similar to that known from wave and plasma observations at Earth (Tsurutani and Rodriguez, 1981 and references therein). The structure of the Earth's foreshock is illustrated in Fig. 1 (Greenstadt et al., 1995). The present paper gives a summary of wave observations performed in the foreshock region at Mars and points out some new features in comparison with observations made in the nearEarth space.

\section{Wave Observations in the Foreshock Region at Mars}

Figure 2 presents typical wave observations in the Martian foreshock carried out by the PWS experiment on 1 March 1989 when the spacecraft was settled on circular orbit $\left(\mathrm{R}=2.8 \mathrm{R}_{\mathrm{M}}\right)$. After the outbound crossing of the Martian bow shock at 19:35 UT the spacecraft successively passed through the ion foreshock, the electron foreshock, the undisturbed solar wind and the inbound quasiperpendicular bow shock. The existence of the ion foreshock at Mars marked by B in Fig. 2 was stated by Grard et al. (1991) and was based on observations of the broadband electric noise upstream the Martian bow shock. This noise, seen in Fig. 2 as short enhancements of signals delivered by filters which cover frequency range from $750 \mathrm{~Hz}$ to $4 \mathrm{kHz}$, and interpreted as the ion acoustic waves, is typically weak in amplitude and bursty in nature. The high frequency emissions measured in the frequency range from 5.8 to $28 \mathrm{kHz}$ have also low intensities in comparison with their amplitudes, which are 


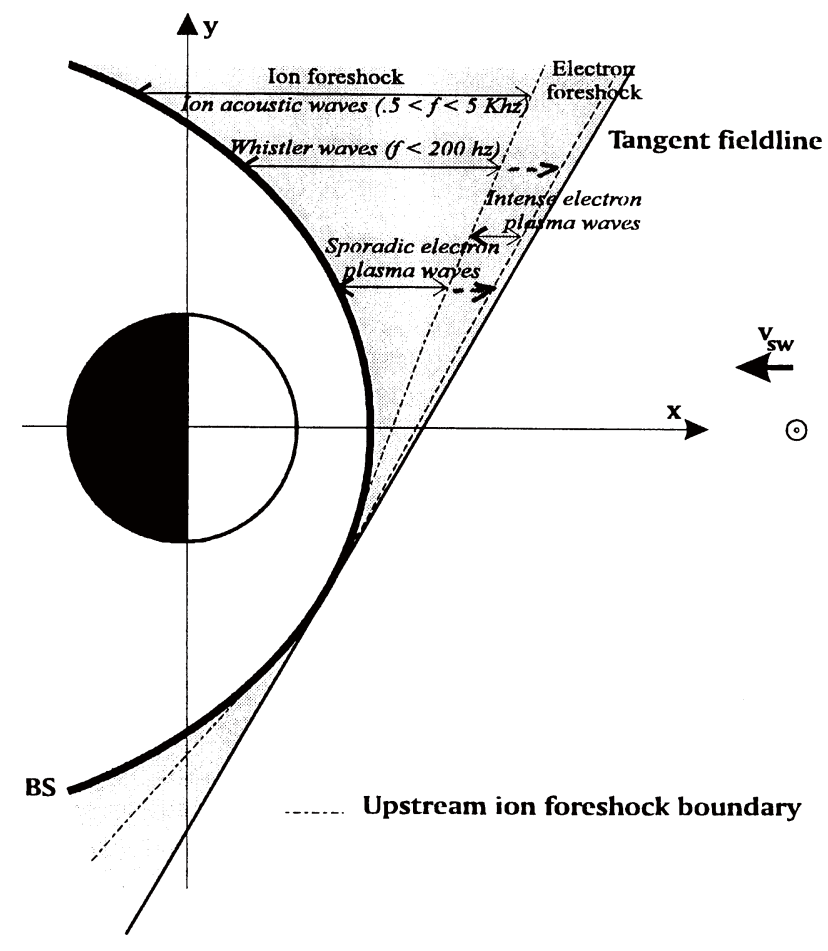

Fig. 1. The summary of wave observations in the Earth's foreshock. Wave modes, which are typical for the electron and ion foreshock are indicated.

observed further, in the electron foreshock. The electron foreshock (region C in Fig. 2) is associated with intense bursts of high frequency waves. It was shown by Skalsky et al. (1992) that the region, where these waves are observed, is adjacent to the magnetic field line which is tangent to the bow shock. The emissions are polarized along the ambient magnetic field and their frequencies are around the electron plasma frequency estimated with simultaneous plasma measurements. The apparent broadening of spectra at high frequencies seen in the electron foreshock in Fig. 2 is entirely instrumental and the natural emissions could be narrow-band ones (Skalsky et al., 1992). The electron measurements carried out simultaneously with observations of high frequency waves have revealed the presence of enhanced fluxes of particles which are streaming back from the bow shock along the magnetic field (Skalsky et al., 1993).

The wave emissions between $10 \mathrm{~Hz}$ and $200 \mathrm{~Hz}$, which are believed to propagate in the whistler mode, are observed in Fig. 2 from 19:35 UT to 20:45 UT. The upstream boundary of their appearance at 20:45 UT is slightly shifted down from that of the electron foreshock. It is distinctly seen in Fig. 2 from the filter bank channels covering frequency bands $50-100 \mathrm{~Hz}$ and $100-150 \mathrm{~Hz}$. The channel $5-50 \mathrm{~Hz}$ seems to be influenced with the ULF emissions due to the broad frequency response of filter bank elements (Grard et al., 1989a; Skalsky et al., 1992). The ULF emissions measured in the frequency range between 0.2 and $10 \mathrm{~Hz}$ gradually increase in their amplitudes through out the entire foreshock region and their maximum intensities are recorded close to the shock front. Tarasov et al. (1998) have shown that the local enhancements of the ULF magnetic oscillations were observed around the proton cyclotron frequency inside the ion foreshock in association with the intense ion fluxes reflected from the Martian bow shock. The high frequency emissions are seen at the leading edge of the shock foot when the spacecraft passed through the region adjacent to the quasiperpendicular bow shock (region E in Fig. 2). Both ULF emissions and broadband electric noise are detected through out the entire foot region. More details on the wave measurements made during the crossing of the quasiperpendicular Martian shock is given elsewhere by Trotignon et al. (1991).

The statistics of wave observations upstream of the Martian bow shock in March 1989 is presented in Fig. 3. The intensities of electric oscillations at different frequencies are shown in this figure as a function of the angle ANG which describes the spacecraft location in the upstream region. The angle ANG is defined as the angle between the line which is the tangent to the bow shock being, at the same time, parallel to the ambient magnetic field, and to the line connecting the tangent point on the bow shock surface with the spacecraft location. By convention, the angle ANG is negative when the spacecraft is not magnetically connected to the bow shock surface and, conversely, is positive when the spacecraft is connected with the bow shock along the magnetic field line. The algorithm used for calculations of the angle ANG is similar to that described by Delva and Dubinin (1998).

It is clearly seen in Fig. 3 that waves in the range of electron plasma frequency (top panel) are the most intense close to the boundary of the electron foreshock (the angle ANG is equal to $0^{\circ}$ or $+/-180^{\circ}$ ). Their maximum amplitudes decrease when the spacecraft penetrates deeper into the foreshock. By contrast, the low frequency emissions measured at frequencies between 0.2 and $10 \mathrm{~Hz}$, gradually increase, for most of the orbits, with depth of the spacecraft location inside the foreshock region. The enhanced level of electric field oscillations in the frequency range from 50 to $200 \mathrm{~Hz}$ is typically detected inside the foreshock when the angle ANG is positive. The intensities of the broadband electric emissions at frequencies between 0.5 and $4 \mathrm{kHz}$ are weak and comparable with the noise level of the instrument which make it difficult to make conclusions on their relation with the spacecraft location inside the foreshock. Nevertheless, it appears that the most intense bursts of these emissions occur deeper in the foreshock region in comparison with wave activities at higher frequencies.

Enhancements of electric signals at frequencies 0.2-10 $\mathrm{Hz}$ and $50-200 \mathrm{~Hz}$ are also seen in Fig. 3 at angles around $-100^{\circ}$. Negative values of angle ANG indicate that these emissions are detected far from the foreshock boundary, in the undisturbed solar wind. It seems that these waves can be of the solar wind origin and are similar to those reported by Neubauer et al. (1977).

\section{Wave Observations at the Boundary of the Electron Foreshock: VLF Emissions Up- stream of It?}

Figure 4(a) presents an example of wave observations on 03 March 1989 when the spacecraft crosses the boundary of the electron foreshock. The crossing of the electron foreshock 


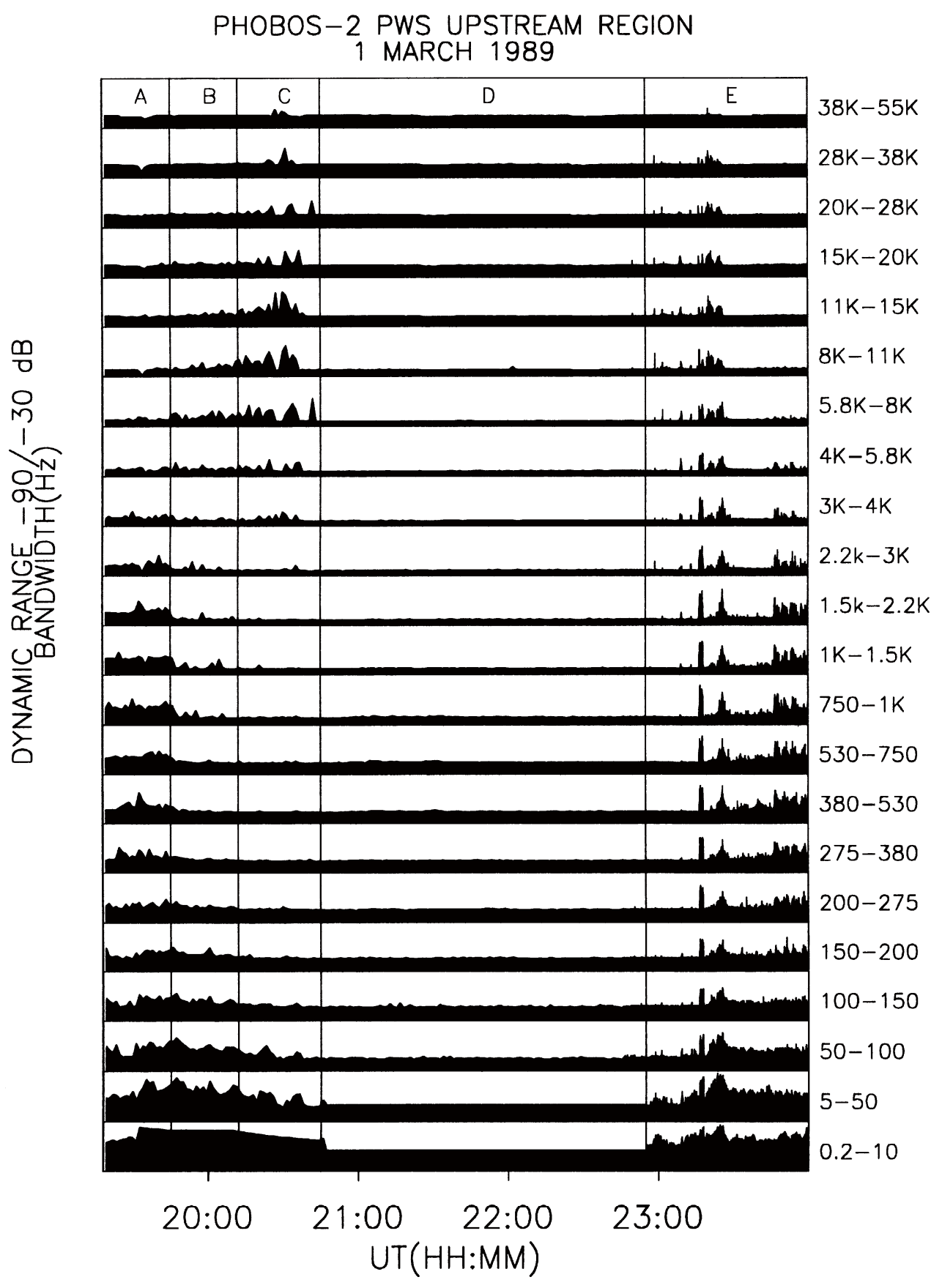

Fig. 2. Wave observations made with the PWS experiment in the upstream region of Mars on 1 March 1989. The signals delivered by 22 elements of the PWS filter bank are shown. The letters in the top panel denote: (A) the magnetosheath, (B) the ion foreshock, (C) the electron foreshock, (D) the undisturbed solar wind and (E) the inbound crossing of the quasiperpendicular bow shock.

boundary is unambiguously identified at 22:53 UT with the onset of high frequency emissions. This is qualitatively confirmed by calculations of the magnetic connection to the bow shock surface. The angle ANG, which describes the spacecraft position with respect to the tangent magnetic field line, changes its sign around this time, i.e. when the spacecraft penetrates the electron foreshock. The ULF fluctuations start to increase simultaneously with the appearance of high frequency waves as it is revealed by statistical analysis given in the previous section. In contrast to the observations on 1 March 1989, waves at frequencies between 50 and $200 \mathrm{~Hz}$ are clearly seen between 22:32 UT22:53 UT in front of the electron foreshock. The outbound crossing of the electron foreshock on 20 February 1989 , shown in Fig. 4(b), is similar to that depicted in Fig. 4(a). Waves at frequencies between 50 and $200 \mathrm{~Hz}$ increase their amplitudes at 03:57 UT upstream the foreshock boundary which is identified at 03:53 UT with the appearance of the high frequency emissions. Unfortunately, magnetic field data are not available for the time period shown in Fig. 4(b) and the crossing of the foreshock boundary could not be supported by calculations of the angle ANG.

Waves seen in Fig. 4(a) between 21:20 UT and 21:40 UT are believed to be emissions of the solar wind origin mentioned in the previous section. The angle ANG, which describes the spacecraft position with respect to the electron 

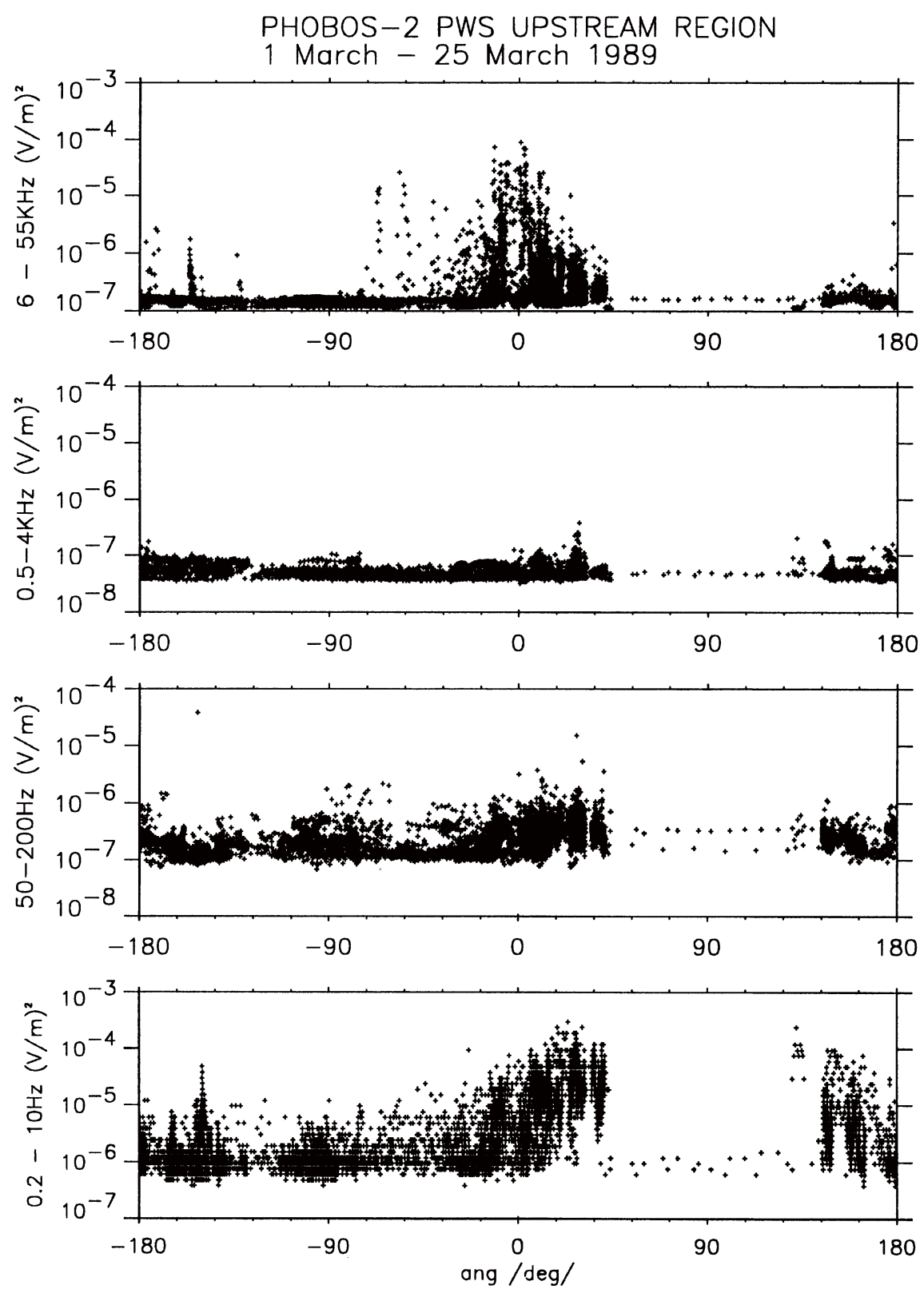

Fig. 3. Statistics of wave observations in March 1989 is given. The square amplitudes of wave intensities in frequency ranges 0-10 Hz, 50-200 Hz, $0.5-4 \mathrm{kHz}$ and $6-55 \mathrm{kHz}$ are shown as a function of angle ANG which describes the spacecraft position in the upstream region with respect to the foreshock boundary (see explanation in text). Negative values of the angle ANG correspond to the spacecraft location outside the foreshock region; positive values indicate that the spacecraft is magnetically connected to the bow shock surface. The gap between $60^{\circ}$ and $130^{\circ}$ seen in all panels corresponds to time intervals when the spacecraft encounters the bow shock and relevant data are not shown.

foreshock boundary occurs between $-90^{\circ}$ and $-150^{\circ}$ for this time interval, i.e. the spacecraft is far from the foreshock, in the solar wind.

The onset of waves at frequencies between 50 and $200 \mathrm{~Hz}$ occurs upstream the electron foreshock boundary identified with the appearance of high frequency emissions, for around $30 \%$ of the Phobos- 2 spacecraft passes through the foreshock region. These events are detected for both inbound and outbound crossings. The frequency range, in which maximum intensity of these emissions are observed, is below or around the electron gyrofrequency for typical values of magnetic field (2-10 nT) at the Mars orbit. However, it is difficult to draw a conclusion on a frequency range of the emissions. Unfortunately, the broad response of the PWS instrument even at the monochromatic input signals does not allow to carry out detailed studies of the bandwidth of natural noises (Skalsky et al., 1992).

\section{Conclusions}

Wave and plasma measurements carried out by the Phobos2 spacecraft near Mars demonstrate formal similarities between physical phenomena ongoing in the foreshock region of both Martian and terrestrial bow shocks. However, waves at frequencies between 50-200 Hz detected upstream the foreshock boundary have been never reported from observation in the near Earth's space. The Martian bow shock is different from another planetary bow shocks because of its finite surface curvature and relatively large foot region. It 


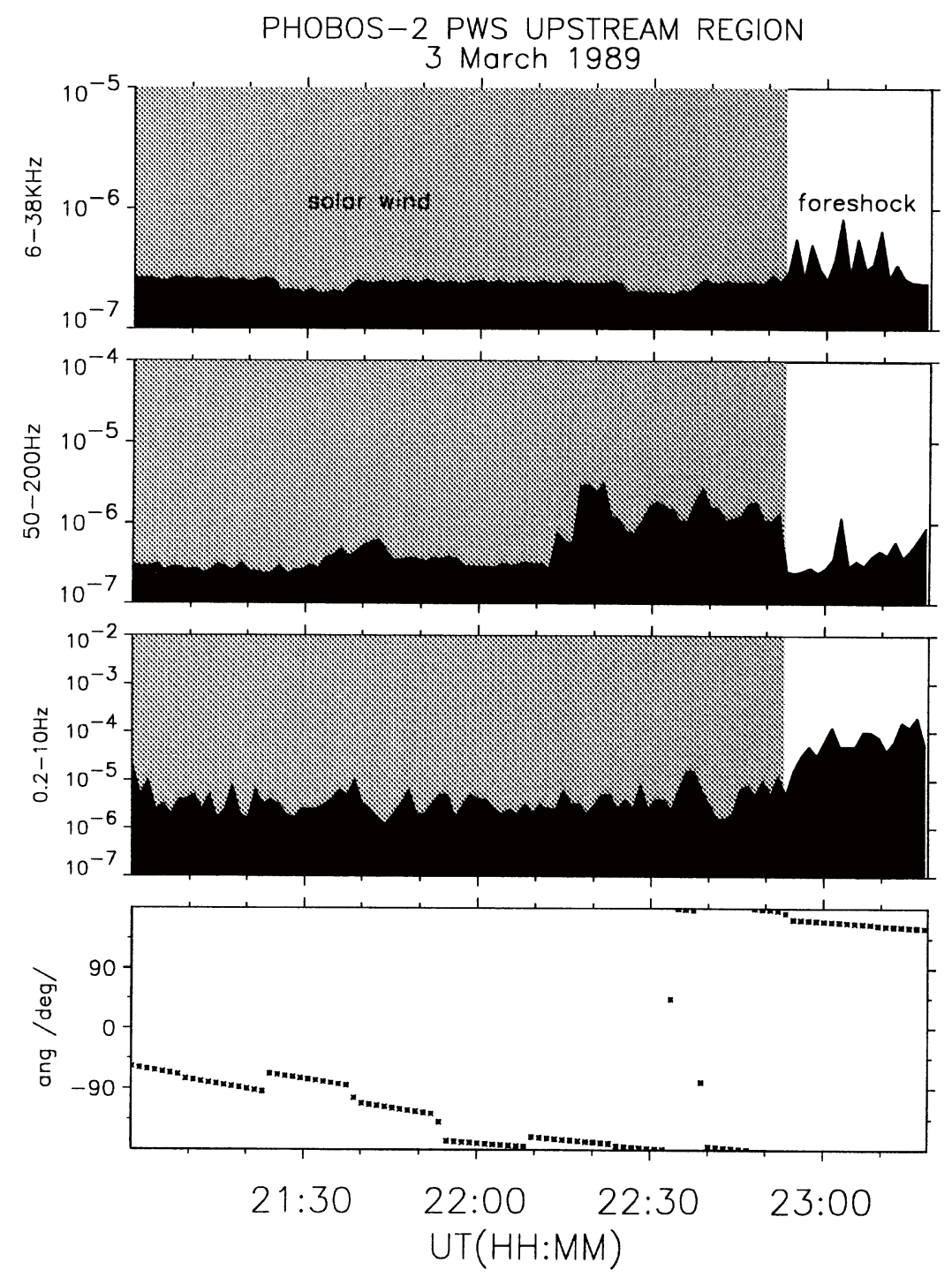

(a)

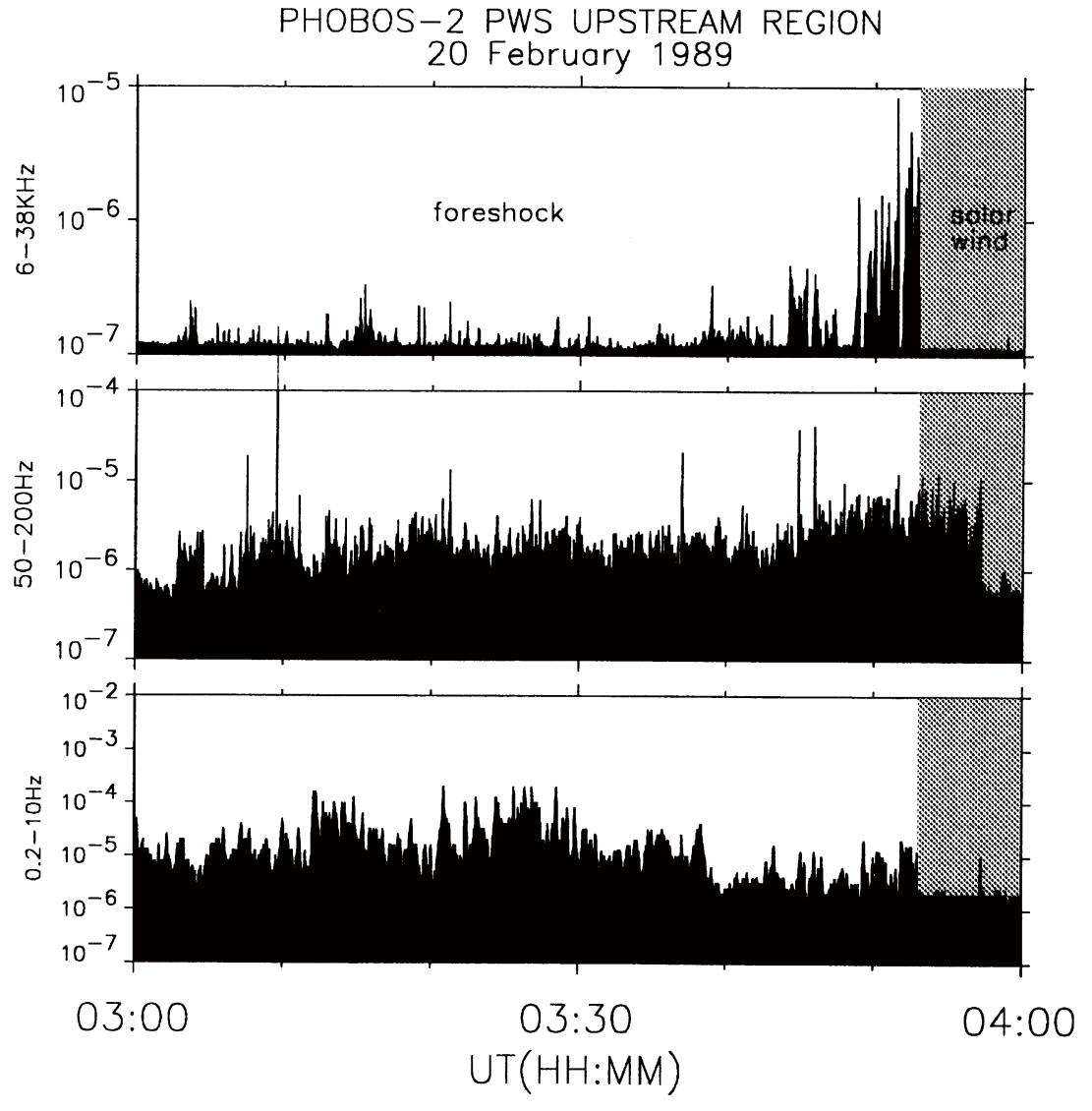

(b)

Fig. 4. Wave observations at the boundary of the electron foreshock made with the PWS experiment on 03 March 1989 (a) and 20 February 1989 (b). (a) Wave intensities expressed in $(\mathrm{V} / \mathrm{m})^{2}$ in the frequency ranges $6-38 \mathrm{kHz}, 50-200 \mathrm{~Hz}$ and $0.2-10 \mathrm{~Hz}$; the angle ANG which describes the spacecraft locations with respect to the foreshock boundary are shown from top to bottom. (b) Wave intensities expressed in $(\mathrm{V} / \mathrm{m})^{2}$ in the frequency ranges 6-38 kHz, 50-200 Hz and $0.2-10 \mathrm{~Hz}$ are shown from top to bottom. 
can be tentatively supposed that waves observed upstream the electron foreshock boundary are linked with this feature of the Martian environment, i.e. these waves are excited in the foot region, which significantly extends beyond the tangent line. However, it cannot be completely excluded that the waves detected upstream the electron foreshock boundary are originated from the solar wind and their coincidence with the crossing of this boundary is occasional. Both issues need more comprehensive analysis of simultaneous plasma, magnetic field and wave coming data, and will be discussed in more details in the further study (Dubinin et al., 1998).

Acknowledgments. The authors wish to express thanks to the ISSI in Bern for the support in the collaboration in the framework of Visiting Science Program. The work of A.Skalsky and S. Klimov was supported by the INTAS grant 94-3626.

\section{References}

Barabash, S. and R. Lundin, Reflected ions near Mars, Phobos-2 observations, Geophys. Res. Lett., 18, 787-790, 1991.

Delva, M. and E. Dubinin, Upstream ULF fluctuations near Mars, $J$. Geophys. Res., 103, 317-326, 1998.

Dubinin, E., D. Obod, A. Pedersen, and R. Grard, Mass-loading asymmetry in the upstream region near Mars, Geophys. Res. Lett., 21, 2769-2772, 1994.

Dubinin, E., D. Obod, R. Lundin, K. Schwingenschuh, and R. Grard, Some features of the Martian bow shock, Adv. Space Res., 15, 8(9)423431, 1995.

Dubinin, E., A. Skalsky, K. Szego, and K. Schwingenschuh, The electron foreshock boundary at Mars, Geophys. Res. Lett., 1998 (to be submitted).

Grard, R., A. Pedersen, S. Klimov, S. Savin, A. Skalsky, J.-G. Trotignon, and C. Kennel, First measurements of plasma waves near Mars, Nature, 341, 607-609, 1989a.

Grard, R., D. Klinge, S. Klimov, S. Savin, and J.-G. Trotignon, The plasma wave system on PHOBOS, J. Phys. E Sci. Instrument, 22, 888, $1989 b$.

Grard, R., C. Nairn, A. Pedersen, S. Klimov, S. Savin, A. Skalsky, and J. -G. Trotignon, Plasma and waves around Mars, Planet. Space Sci., 39, 89-98, 1991.

Greenstadt, E., G. Le, and R. Strangeway, ULF waves in the foreshock, Adv. Space Res., 15, 8(9)71, 1995.

Kiraly, P., R. Loch, K. Szego, I. Szemerey, I. T. Szucs, M. Tatrallyay, N. Shutte, A. Dyachkov, K. Gringauz, S. Sheronova, and M. Verigin, The HARP plasma experiment on-board the Phobos-2 spacecraft: preliminary results, Planet. Space Sci., 39, 139-145, 1991.

Neubauer, F. M., H. Beinroth, H. Barnstorf, and G. Dehmel, Initial results from the Helios 1 search coil magnetometer experiment, J. Geophys. Res., 82, 599-605, 1977.

Russell, C., J. G. Lumann, K. Schwingenschuh, W. Riedler, and Ye. Yeroshenko, Upstream waves at Mars: Phobos observations, Geophys. Res. Lett., 17, 897-900, 1990.

Skalsky, A., R. Grard, C. M. C. Nairn, S. Klimov, J.-G. Trotignon, and K. Schwingenshuh, The Martian bow shock: wave observations in the upstream region, J. Geophys. Res., 92, 2927-2933, 1992.

Skalsky, A., R. Grard, P. Kiraly, S. Klimov, V. Kopanyi, K. Schwingenschuh, and J.-G. Trotignon, Simultaneous plasma wave and electron flux observations upstream of the Martian bow shock, Planet. Space Sci., 41, 183-188, 1993.

Tarasov, V., E. Dubinin, S. Perraut, K. Sauer, and A. Skalsky, Wavelet application to the magnetic field turbulence in the upstream of the Martian bow shock, Earth Planets Space, 50, 1998 (in press).

Trotignon, J.-G., M. Hamelin, R. Grard, A. Pedresen, S. Klimov, S. Savin, A. Skalsky, and C. Kennel, A comparison between the Earth's and Mars' bow shocks detected by the PHOBOS PLASMA-WAVE system, Planet. Space Sci., 39, 99-112, 1991.

Tsurutani, B. and P. Rodriguez, Upstream waves and particles: An overview of ISEE results, J. Geophys. Res., 86, 4319-4324, 1981.

A. Skalsky (e-mail: askalsky@vm1.iki.rssi.ru), E. Dubinin (e-mail: dubinin@helene.mpae.gwdg.de), M. Delva (e-mail: delva@fiwf01.tugraz.ac.at), R. Grard, S. Klimov, K. Sauer (e-mail: sauer@linax1.mpae.gwdg.de), and J.-G.Trotignon (e-mail:jgtrotig@cnrsorleans.fr) 